\title{
Highly Sensitive Coherent anti-Stokes Raman Scattering Im- aging of Protein Crystals
}

Grigory M. Arzumanyan ${ }^{*},{ }^{+\neq}$Nelya V. Doroshkevich, ${ }^{\dagger}$ Kahramon Z. Mamatkulov, ${ }^{\dagger}$ Sergey N. Shashkov, ${ }^{\S}$ Egor V. Zinovev, ${ }^{\perp}$ Alexey V. Vlasov, ${ }^{\perp}$ Ekaterina S. Round ${ }^{\perp, \|, \nabla}$ and Valentin I. Gordeliy ${ }^{*},{ }^{\perp} \|, \nabla$

${ }^{\dagger}$ Joint Institute for Nuclear Research, 141980 Dubna, Russia

${ }^{\ddagger}$ Dubna State University, 19 Universitetskaya st., Dubna, 141982, Russia

${ }^{\S}$ SOL Instruments, 220005 BY P.O. Box 235, Minsk, Republic of Belarus

${ }^{\perp}$ Moscow Institute of Physics and Technology, 141700 Dolgoprudny, Moscow region, Russia

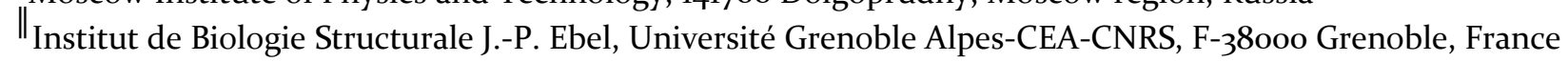

${ }^{\nabla}$ Institute of Complex Systems: Structural Biochemistry (ICS-6), Research Centre Jülich, 52425 Jülich, Germany

Supporting Information

\section{Supporting Information}

This section includes detailed information on: 1) expression and purification of bacteriorphodopsin, 2) nanovolume crystallization of bacteriorhodopsin, 3) crystallization of lysozyme, 4) CARS technique in application to protein crystals

\section{Expression and purification of bacteriorhodopsin.}

The expression, solubilization and purification of bR have been previously described in detail'. The protein was cultivated in Halobacterium Salinarum using the peptone medium: firstly, we diluted a fresh starter culture in the latelog phase 1:50 in peptone medium and cultivated it at the optimal temperature of $37-40^{\circ} \mathrm{C}$. Then we shook the cultures with $150 \mathrm{rpm} / \mathrm{min}$ in rotary shakers with the motion of $\varnothing 50 \mathrm{~mm}$. To promote increased expression of bacteriorhodopsin, the oxygen concentration was reduced in the culture when reaching midlog phase after approx 2-4 days of vigorous shaking. Next, the shaking motion was changed to 80 rpm to decrease aeration. The final step is harvesting cells by centrifugation after $4^{-6}$ days. Lysis was made with washing the pelleted cells in basal salt solution, resuspending $1 \mathrm{~g}$ of the wet cell pellet in $25 \mathrm{~mL}$ 0.02\% $\mathrm{NaN}_{3}$ in $\mathrm{H}_{2} \mathrm{O}$ and adding DNAse I (approx. $5 \mathrm{mg} / \mathrm{ml}$ suspension). Purple membranes (PMs) were separated from cell debris and other impurities with a series of centrifugation steps. 
To solubilize PMs we diluted PM suspension with a solubilization buffer to the final bR concentration of $2.5 \mathrm{mg} / \mathrm{ml}$, stirred the suspension $2 \mathrm{~d}$ at $4^{\circ} \mathrm{C}$, and spinned the sample at goooog in a centrifuge for $1 \mathrm{~h}$ at $4^{\circ} \mathrm{C}$. Then we added 2.125 parts of the crystallization buffer to the bR solution to replace the solubilization buffer.

\section{Nanovolume crystallization of $b R$.}

High quality crystals were grown in a lipidic phase of monoolein (MO) in accordance with our protocols. ${ }^{1,2}$

$\mathrm{MO}$ was melted at $\sim 40^{\circ} \mathrm{C}$ during 20 min and then mixed with a buffer solution using two gas-tight syringes (Hamilton, USA). ${ }^{3}$ The resulting phase was then mixed with the BR concentrated at $30 \mathrm{~g} \cdot \mathrm{L}^{-1}$ in different volume ratios by two gas-tight syringes used for crystallization trials. Dispensing the phases by 100-nL drops on the 96-well lipidic cubic phase (LCP) Sandwich Set glass plates (Marienfeld, Germany) and adding $800 \mathrm{~nL}$ of the precipitant solution to each drop of the lipidic mesophase was performed by an NT8 Formulatrix (Waltham, MA) crystallization robot. $\mathrm{Na} / \mathrm{K}-\mathrm{P}_{\mathrm{i}}, \mathrm{pH} 5.6$ solutions at concentrations between $0.8-2 \mathrm{M}$ were used as precipitants. Hexagonal plate-like bR crystals of $\mathrm{P}_{3}$ symmetry were observed within 7-21 days after the precipitant was added. The lipidic mesophase that yielded the well diffracting crystals was prepared by mixing MO, buffer, and the $30-\mathrm{g} \cdot \mathrm{L}^{-1}$ bR solution. CARS and SHG imaging of the crystals was done directly with the glass plates. To verify the potential of CARS in imaging of merohedrally twinned crystals we used earlier developed protocols of bR crystallization known to result in the growth of merohedrally twinned crystals..$^{4-6}$

\section{Crystallization of Lysozyme.}

Lysozyme from AppliChem (LOT 8Ro11640) was crystallized using hanging drop and batch LCP techniques. For the hanging drop method we used the precipitant, containing $\mathrm{NaCl}$ (from o.6M to $1.6 \mathrm{M}$ ) and Sodium Acetate (o.1M, $\mathrm{pH}$ from 4.0 to 4.9). ${ }^{7}$ Standard 24-well hanging drop crystallization plates were used. We added $2 \mu \mathrm{l}$ of the precipitant to $2 \mu \mathrm{l}$ of $50 \mathrm{mg} / \mathrm{ml}$ Lysozyme in o.o2M Sodium Acetate $\mathrm{pH}$ 4.9. Reservoir was filled with 50opl of the precipitant solution. LCP crystallization of lysozyme was made according to ${ }^{8}$.

IV. Laser-scanning CARS technique used for imaging of protein crystals.

The sample is stimulated through a four-wave mixing process (Figure S1). ${ }^{9-11}$ The multimodal optical platform for performing Raman, CARS, SHG, and transmitted light imaging where all experiments were done is shown in Figure S2. It's well-known that the pulse duration of several 
picoseconds is a proper compromise between high intensity and narrow spectral bandwidth necessary for the CARS microscopy. ${ }^{12}$ Besides, its intensity is sufficient also for detection of other nonlinear processes, in particular the second and sum harmonic generation. Thus, a picosecond $\mathrm{Nd}: \mathrm{YVO}_{4}$ tunable laser (EKSPLA, PT257-SOPO, Lithuania) with a pulse width of $\sim 6 \mathrm{ps}$ and a repetition rate of $85 \mathrm{MHz}$ is used as the source of the Stokes wave $\left(\omega_{\mathrm{s}}\right)$ and is simultaneously used to synchronously pump an intracavity-doubled crystal optical parametric oscillator (SOPO). Thereby, the SOPO coherent device provides temporal synchronization with the $\mathrm{Nd}: \mathrm{YVO}_{4}$ and serves as a source of the pump beam $\left(\omega_{\mathrm{p}}\right)$ tunable from $690 \mathrm{~nm}$ to $990 \mathrm{~nm}$ with a maximum output power of $200 \mathrm{~mW}$ with a nearly Fourier transform-limited linewidth $\left(\sim 6 \mathrm{~cm}^{-1}\right)$. Only a small portion of biologically tolerable laser power is used for CARS and SHG imaging. We exploit parallel orientations of linear polarizations of the input Stokes and pump beams. Both excitation picosecond pulse trains are made coincident in time and in space utilizing an optical delay line and a series of dichroic mirrors. For CARS microscopy, we use a waterimmersion objective lens with a high numerical aperture (NA=1.2, UPLANAPO-6ox, Olympus) to focus the beams tightly. With the tight foci, the phase-matching conditions are relaxed because of the large cone of wave vectors of the excitation beams and the short interaction length.

Using our optical platform, a sample can be imaged by utilizing vibration frequencies in the spectral range of (1000-3580) $\mathrm{cm}^{-1}$, which covers all most important vibrational modes of biomolecules. Five detection channels allow two forward- and three backward- propagated signals to be recorded. The anti-Stokes signal at $803 \mathrm{~nm}$, generated in the forward direction (F-CARS), is collected by a condenser lens, spectrally separated from the excitation beams using 794/160 band-pass filter (Semrock, FFo1-794/160-25) and through the Glan-Taylor polarizer installed on a rotation mount directed to the photomultiplier (Hamamatsu, H6780-01). The polarization control is adjustable with a half-wave plate in the Stokes beam.

A computer-controlled XY galvanometer scanner (GSI-Lumonics VMiooo) provided a fast scan of the sample in the lateral focal plane of the objective. All the CARS and SHG images presented in this paper are composed of $500 x 500$ pixels (250,000 pixels) taken by raster scanning the sample at room temperature. The corresponding bars are mentioned in the figures as well. Signal integration time was $3 \mu \mathrm{s} /$ pixel resulting in a total acquisition time of $3 \mathrm{~s}$ (including an image transfer time and an image visualization time). The objective lens is mounted on a com- 
puter-controlled z-axis translation piezostage for scanning through the microscope's optical axis with a minimal scan step of $0.1 \mu \mathrm{m}$.

As for the SHG imaging we exploit the same PMT for detection of P-CARS signals (see Fig.2). The Stokes wave served for second harmonic generation (532 nm) in BR membrane protein crystals and a narrow bandpass filter (Semrock, FFo1-532/3-25) for a SHG signal filtration and further detection on the PMT. Though the detected BR images in SHG are also of high quality and contrast, one should take into account that CARS microscopy is intrinsically more informative than SHG microscopy as it contains information about molecular vibration.

Along with the CARS and SHG signals, the system allows detection of spontaneous Raman (excitations at $633 \mathrm{~nm}$ and $785 \mathrm{~nm}$ ), luminescence (including up-conversion luminescence), second and sum frequency generation signals and transmission mode as well.

\section{References}

(1) Gordeliy, V. I.; Schlesinger, R.; Efremov, R.; Büldt, G.; Heberle, J. Methods in molecular biology (Clifton, N.J.) 2003, 228, 305-16.

(2) Borshchevskiy, V.; Moiseeva, E.; Kuklin, A.; Büldt, G.; Hato, M.; Gordeliy, V. J. Cryst. Growth 2010, 312, 3326-3330.

(3) Cheng, A.; Hummel, B.; Qiu, H.; Caffrey, M. Chem. Phys. Lipids 1998, 95, 11-21.

(4) Borshchevskiy, V.; Efremov, R.; Moiseeva, E.; Büldt, G.; Gordeliy, V. Acta Crystallogr. D. Biol. Crystallogr. 2010, 66, 26-32.

Efremov, R.; Moukhametzianov, R.; Büldt, G.; Gordeliy, V. Biophys. J. 2004, 87, 3608-13.

(5) Borshchevskiy, V.; Gordeliy, V., Modern Aspects of Bulk Crystal and Thin Film Preparation; InTech: Rijeka, 2012, pp 477-496.

(6) Dessau, M. A.; Modis, Y. J. Vis. Exp. 2011, 47, e2285.

(7) Aherne, M.; Lyons, J. A.; Caffrey, M. J. Appl. Crystallogr. 2012, 45, 1330-1333.

(8) Duncan, M. D.; Reintjes, J.; Manuccia, T. J. Opt. Lett. 1982, 7, 350.

(9) Cheng, J.-X.; Xie, X. S. J. Phys. Chem. B 2004, 108, 827-840.

(10) Cheng, J.-X. Appl. Spectrosc. 2007, 61, 197-208.

(11) Haupert, L. M.; Simpson, G. J. Methods 2011, 55, 379-86.

(12) Cheng, J.-X.; Jia, Y. K.; Zheng, G.; Xie, X. S. Biophys. J. 2oo2, 83, 502-9.

(13) Cheng, J.-X.; Book, L. D.; Xie, X. S. Opt. Lett. 2oo1, 26, 1341.

(14) Volkmer, A.; Cheng, J.-X.; Sunney Xie, X. Phys. Rev. Lett. 2oo1, 87, 023901.

(15) Kissick, D. J.; Wanapun, D.; Simpson, G. J. Annu. Rev. Anal. Chem. (Palo Alto. Calif) 2011, 4, 419-37. 


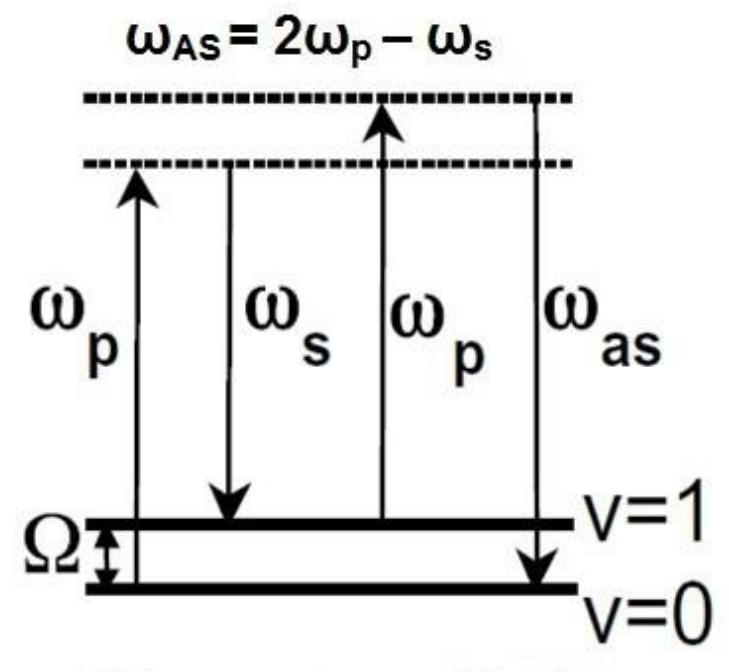

a) Resonant contribution

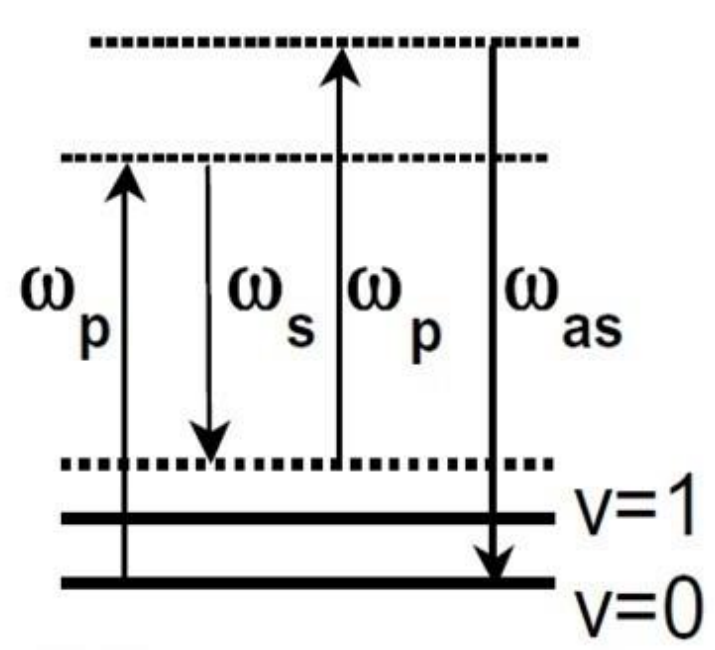

b) Nonresonant contribution

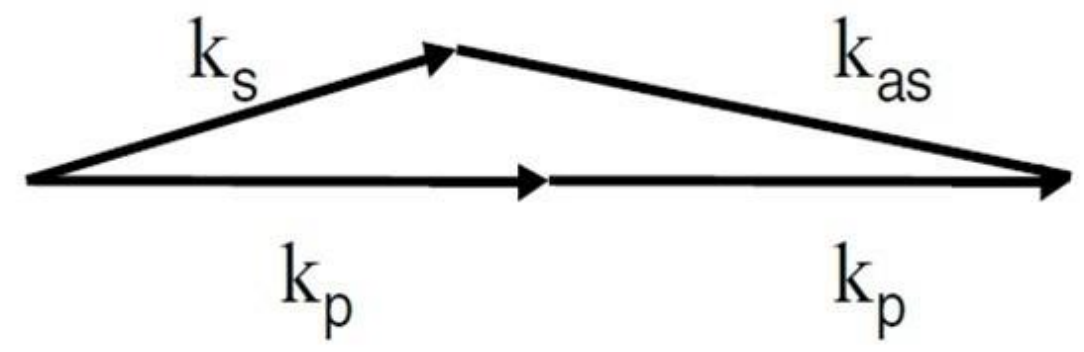

c) Phase matching condition

Figure S1. Energy diagram of CARS. $\omega_{\mathrm{p}}$ is the pump beam frequency, $\omega_{\mathrm{s}}$ is a Stokes beam frequency, $\omega_{\mathrm{as}}=2 \omega_{\mathrm{p}}-\omega$ is a CARS signal at the anti-Stokes frequency. $\mathrm{k}_{\mathrm{p}}, \mathrm{k}_{\mathrm{s}}$ and $\mathrm{k}_{\mathrm{as}}$ are corresponding wave vectors. 


\section{Diagram of the CARS microscope}

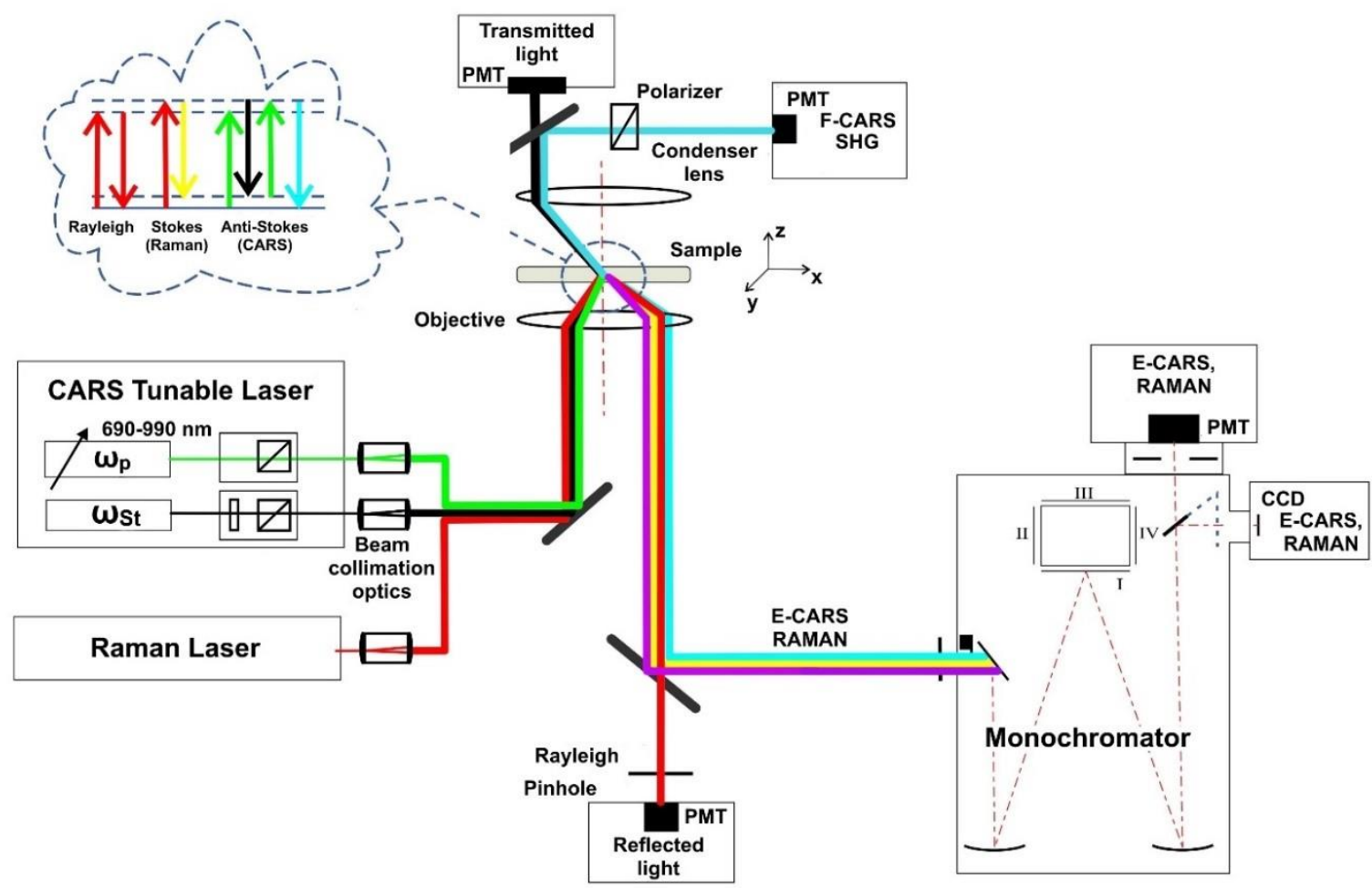

Figure S2. Diagram of the multimodal optical platform: "CARS" microscope. The figure is a schematic illustration of the optical platform. In the experiments the whole aperture of the $\mathrm{NA}=1.2$ objective was used. In all the experiments (not in the scheme) the beams are passing through the center of the objective covering the whole aperture. 


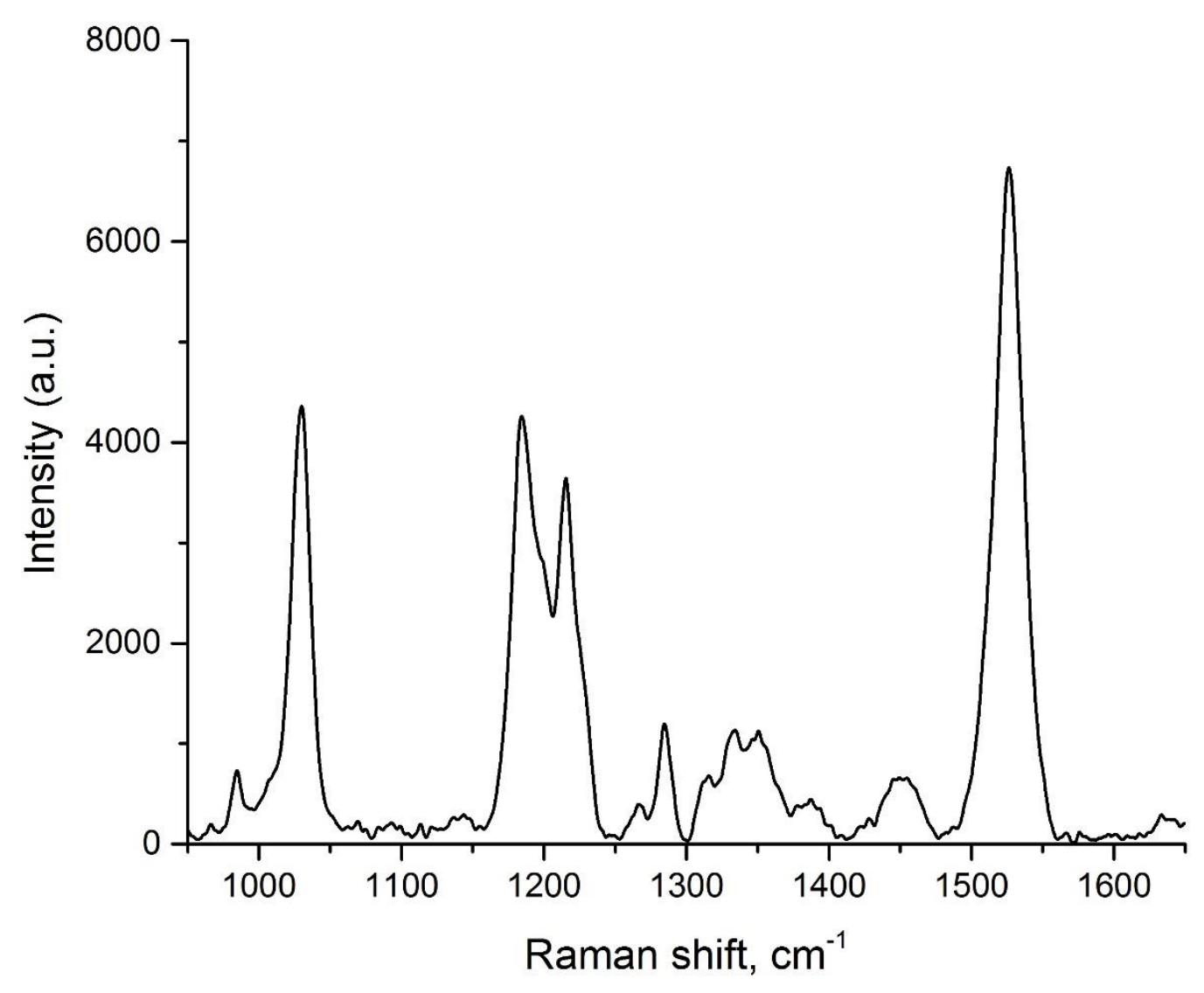

Figure S3. Raman spectrum of bR crystal (excitation $785 \mathrm{~nm}$ ). Raman bands at $1529 \mathrm{~cm}^{-1}$ and $1570 \mathrm{~cm}^{-1}$ were used in CARS studies.

The analytical capabilities of the spontaneous Raman scattering microscopy are significantly limited by the difficulty in acquiring low signals and, as a result of it, a two dimensional Raman map can take a long time.

The Anti-Stokes CARS signal has frequency exceeding pump and Stokes frequencies and is detected in a spectral range free from the stray light of Stokes luminescence. However, the sensitivity of the forward CARS (F-CARS) signal was limited by a nonresonant background which may overshadow weak signals that are of interest. This nonresonant signal can be considered as the result of several far off-resonance transitions that also add coherently. The resonant amplitude contains a phase shift of $\pi$ radians over the resonance whereas the nonresonant part does not. The resonant signal can be separated from the nonresonant background when polarization 
sensitive detection is employed. The P-CARS method improves the image quality and contrast by eliminating the nonresonant background..$^{10,13-16}$
a) F-CARS, $1529 \mathrm{~cm}^{-1}$
b) F-CARS, $1570 \mathrm{~cm}^{-1}$
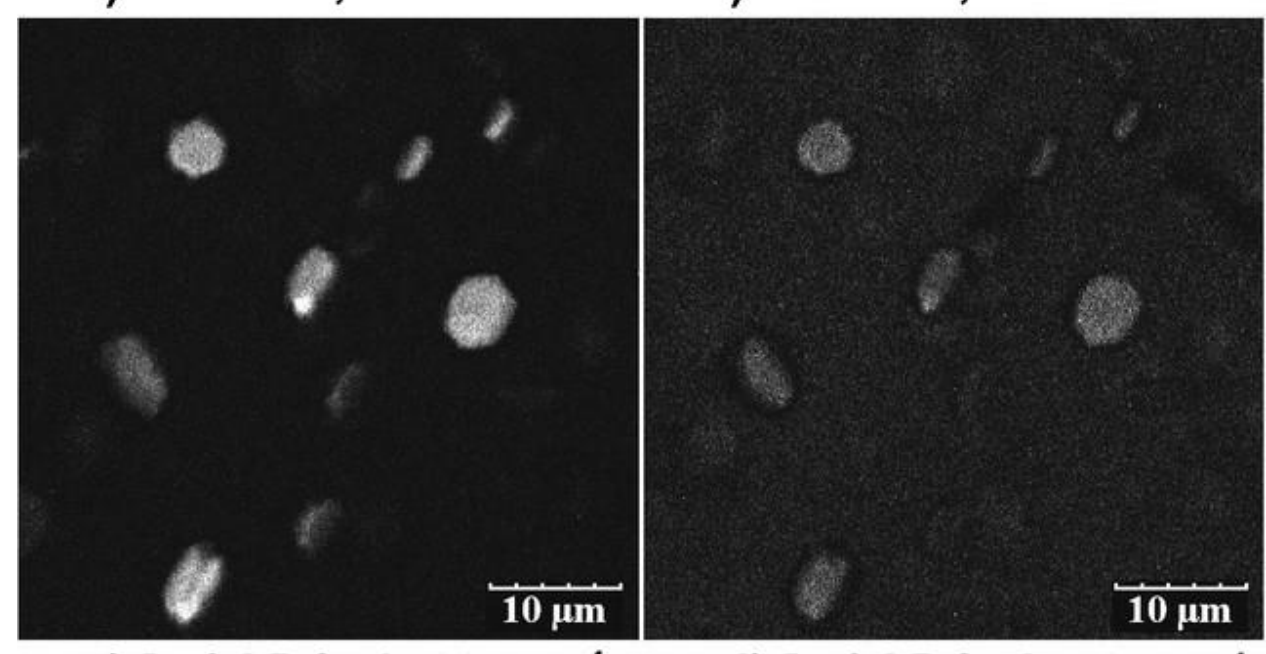

c) P-CARS, $1529 \mathrm{~cm}^{-1}$

d) P-CARS, $1570 \mathrm{~cm}^{-1}$
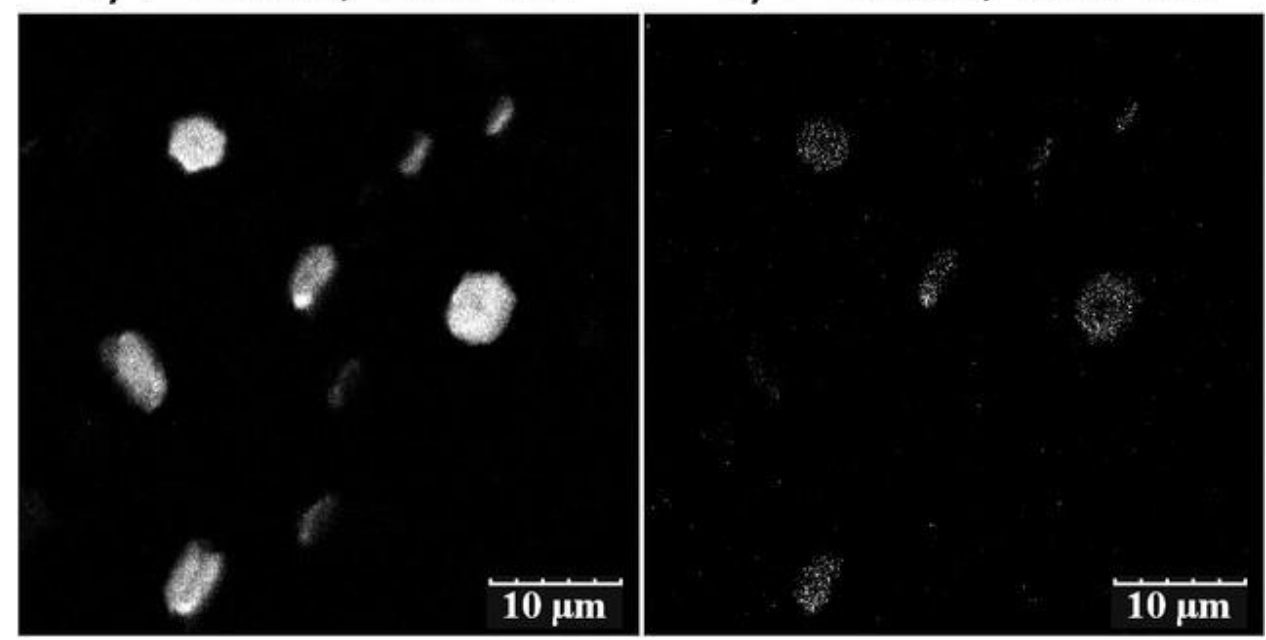

Figure S4. F- and P-CARS images of bacteriorhodopsin crystals corresponding to Raman bands at $1529 \mathrm{~cm}^{-1}$ and $1570 \mathrm{~cm}^{-1}$ (excitation wavelength is $785 \mathrm{~nm}$ ). 
Micrograph

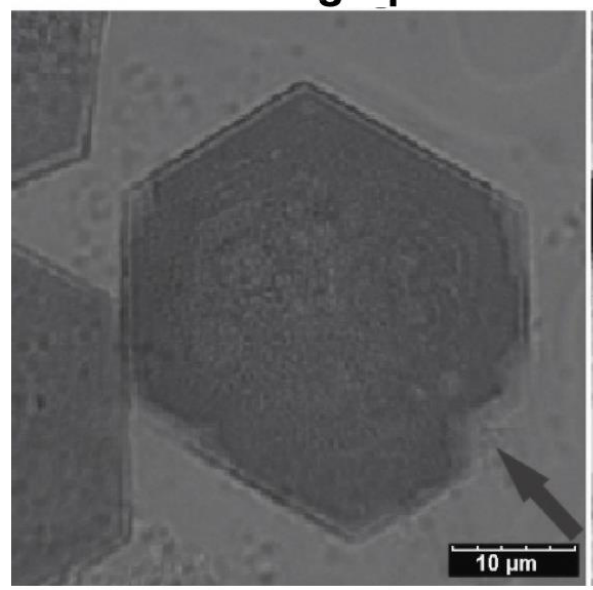

Raman

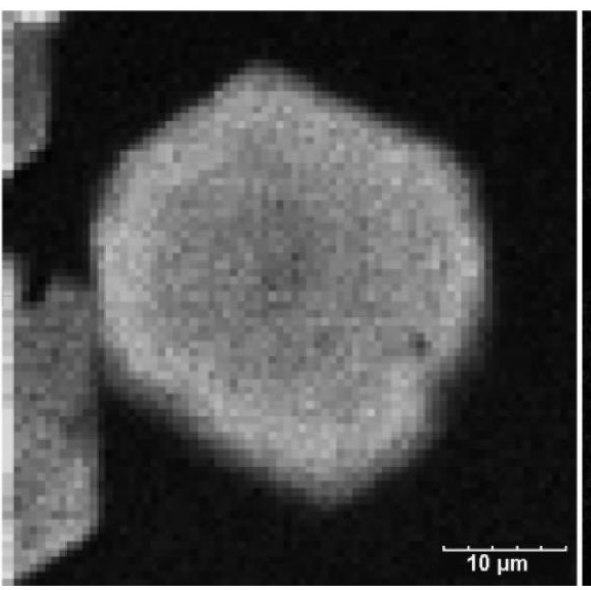

CARS

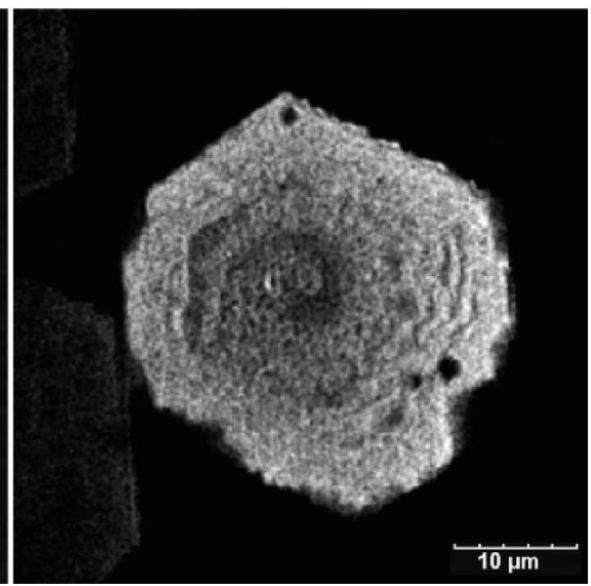

Figure $S_{5}$. A micrograph, Raman $\left(\lambda_{\mathrm{ex}}=785 \mathrm{~nm}\right)$ and P-CARS $\left(\lambda_{\mathrm{p}}=915.5 \mathrm{~nm}, \lambda_{\mathrm{st}}=1064 \mathrm{~nm}\right)$, images at $1529 \mathrm{~cm}^{-1}$ of a bacteriorhodopsin crystal with the in-plane size of $34 \mu \mathrm{m}$. The scan area $-48 \times 48$ $\mu \mathrm{m}^{2}$. The defects of crystal growth are well identified. The optical microscope image gives an impression that the crystal is perfect with the only visible edge defect (it is shown with an arrow). The same conclusion can be made with the Raman image. The analytical capabilities of the spontaneous Raman scattering microscopy are significantly limited by the difficulty in acquiring low signals and, as a result of it, a two dimensional Raman imaging can take a long time. In contrast, CARS resolves tiny features of bR morphology (Figure S6). and displays several considerable defects visible as dark parts of the crystal, the edges of the crystal are not smooth. It is interesting that the optical microscopy image shows weak inhomogeneity at the places were the CARS image of the crystals is dark but the conclusions about their meaning are impossible. An additional feature of the CARS image is that the stair-like morphology of the surface of the crystal is well defined. This type of crystal growth has been described. However, for the case of membrane proteins this has been achieved for the first time with estimated resolution better than $1 \mu \mathrm{m}$. Furthermore the CARS imaging technique is fast compared to other such imaging techniques. 

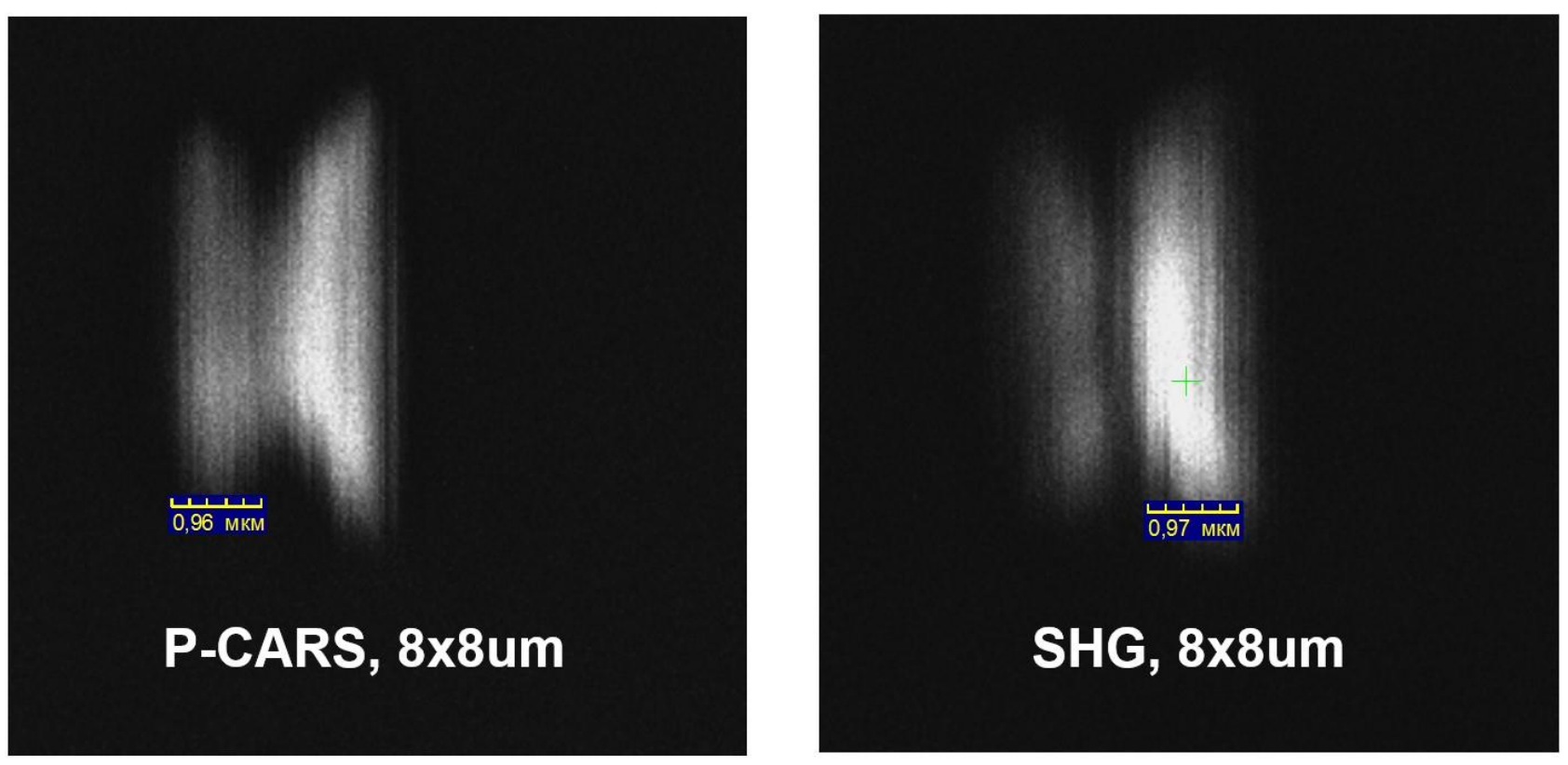

Figure S6. Comparative images of bR microcrystals made by P-CARS and SHG.
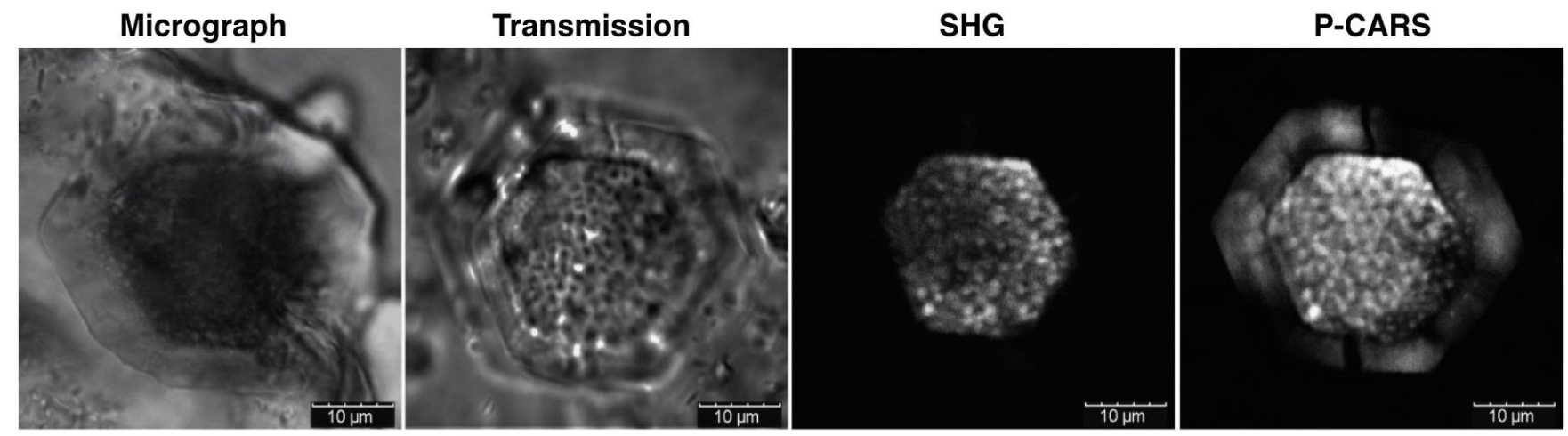

Figure S7. Micrograph, transmission, SHG and P-CARS images of a twin bR crystal with the sizes $20 \times 20 x 2 \mu \mathrm{m}^{3}$ are shown. Two first images (micrograph, transmission) show a belt surrounding the hexagonal crystal. This can be interpreted as a defect of the crystal. Unexpectedly, SHG fails to display any signs of the belt. In contrast, CARS resolves the bR crystalline belt around the main body of the crystal well. To explain the SHG image we suggest that the belt is not a well ordered part of the crystal. Indeed, SHG signal is very strong only in the case of well-ordered crystals. ${ }^{4}$ 

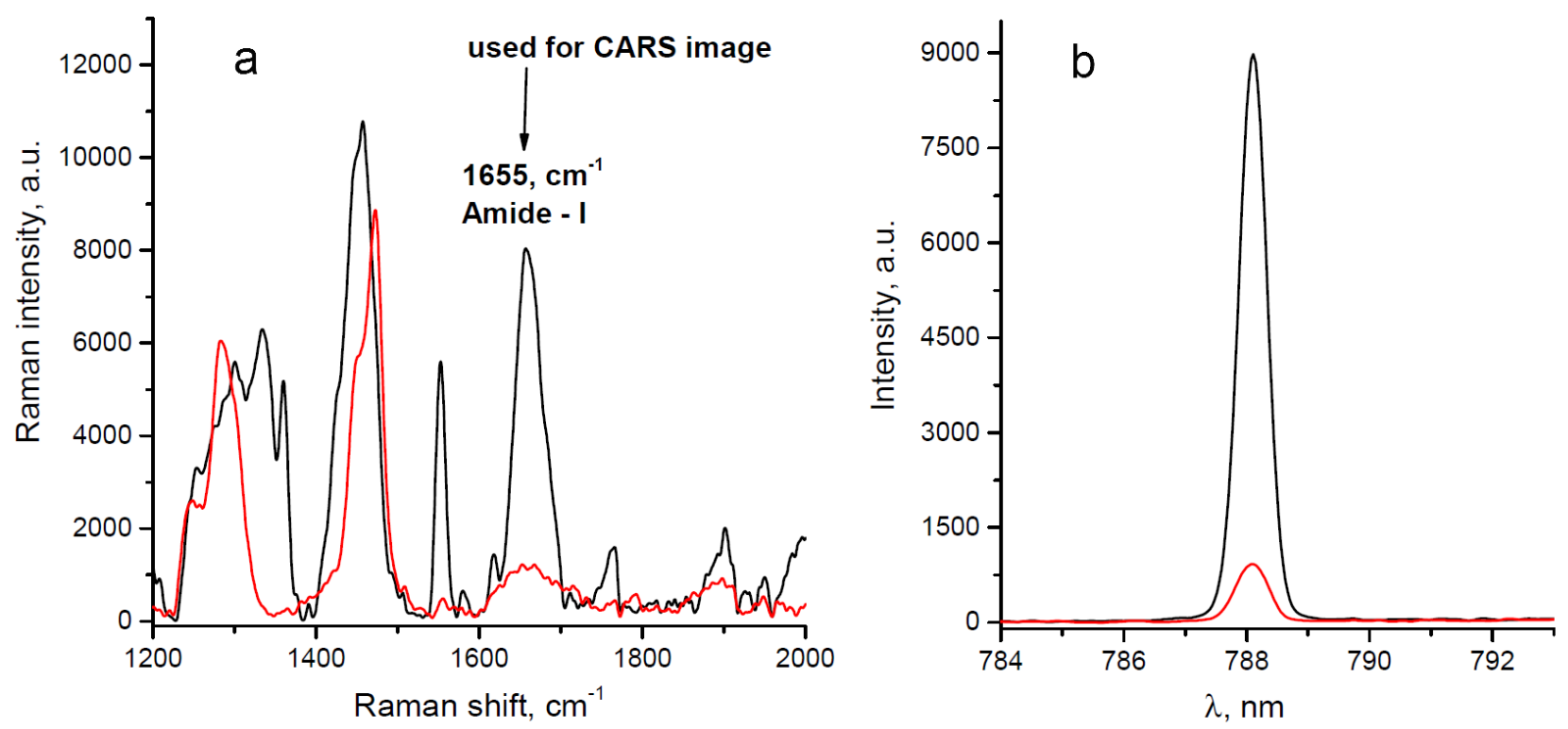

Figure S8. Raman(a) and CARS(b) spectra of lysozyme at a crystal (black) and out of the crystal (red).

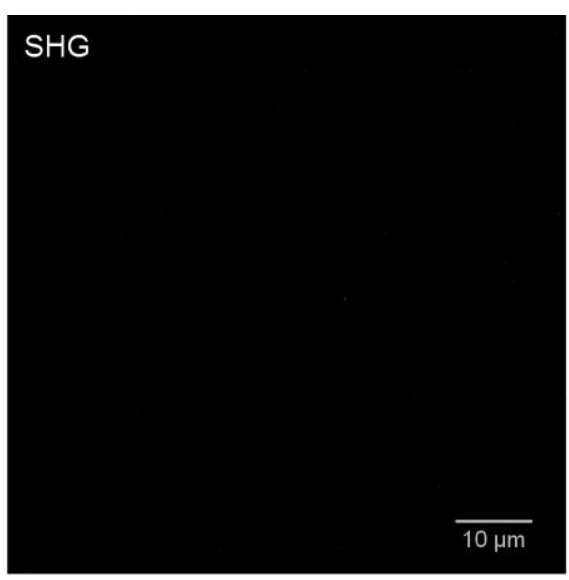

$72.5 \times 72.5 \mu \mathrm{m}$

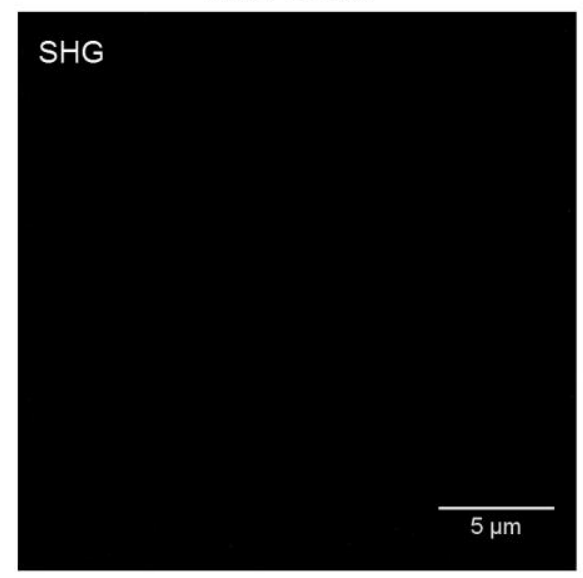

$24 \times 24 \mu \mathrm{m}$

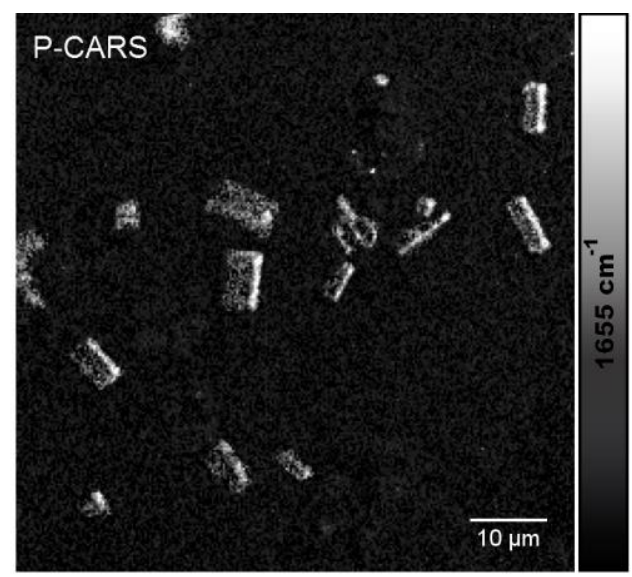

$72.5 \times 72.5 \mu \mathrm{m}$

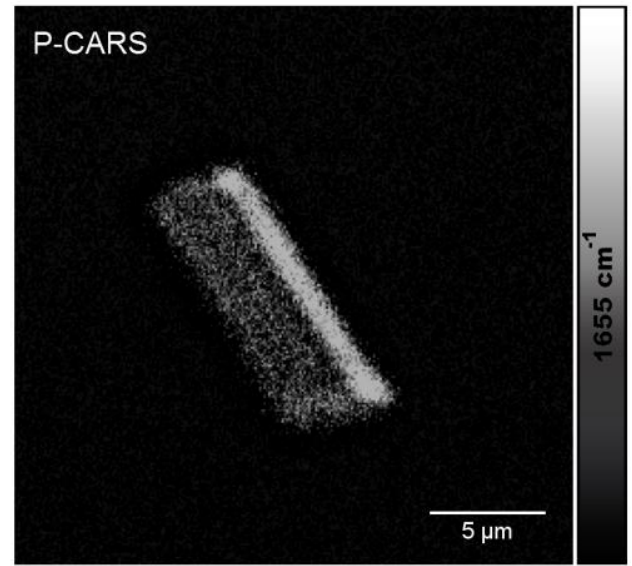

$24 \times 24 \mu \mathrm{m}$

Figure S9. P-CARS images of lysozyme crystals at Amide I $\left(1655 \mathrm{~cm}^{-1}\right)$ band. 


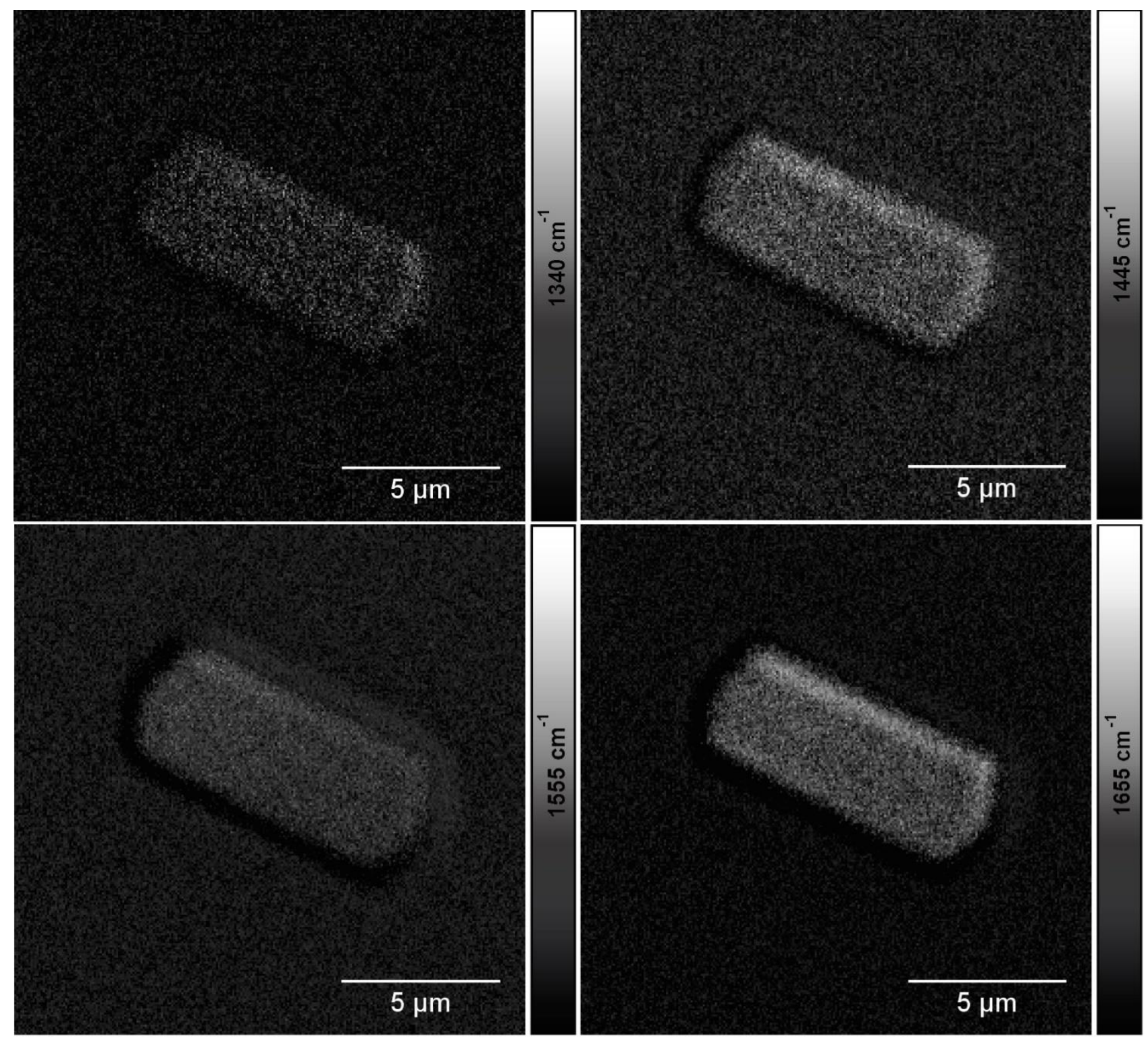

Figure S1o. P-CARS images of lysozyme crystals at Amide III $\left(1340 \mathrm{~cm}^{-1}\right), \mathrm{CH}_{2}\left(1445 \mathrm{~cm}^{-1}\right)$, Amide II $\left(1555 \mathrm{~cm}^{-1}\right)$ and at Amide I $\left(1655 \mathrm{~cm}^{-1}\right)$ bands. 